\title{
AUTOMATIC EXTRACTION OF POWER LINES FROM UAV LIDAR POINT CLOUDS USING A NOVEL SPATIAL FEATURE
}

\author{
M. Zhou ${ }^{1}$, K. Y. Li $^{1,2}$, J. H. Wang ${ }^{1}$, C. R. Li ${ }^{1}{ }^{*}$, G. E. Teng ${ }^{1}$, L. Ma ${ }^{1}$, H. H. Wu ${ }^{1}$, W. Li ${ }^{1}$, H. J. Zhang ${ }^{1}$, J. Y. Chen ${ }^{1}$, L. S. Chen ${ }^{1,2}$ \\ ${ }^{1}$ Key Laboratory of Quantitative Remote Sensing Information Technology, Academy of Opto-Electronics, Chinese Academy of \\ Sciences, Beijing 100094, China - (zhoumei, tenggeer, malian, hhwu, liwei, hjzhang, jychen)@aoe.ac.cn \\ ${ }^{2}$ University of Chinese Academy of Sciences, Beijing 100049, China - (likuangyu15, chenlinsheng16)@mails.ucas.ac.cn
}

KEY WORDS: Power Line Extraction, UAV LiDAR, 3D Spatial Features, Linear Elements

\begin{abstract}
:
UAV LiDAR systems have unique advantage in acquiring 3D geo-information of the targets and the expenses are very reasonable; therefore, they are capable of security inspection of high-voltage power lines. There are already several methods for power line extraction from LiDAR point cloud data. However, the existing methods either introduce classification errors during point cloud filtering, or occasionally unable to detect multiple power lines in vertical arrangement. This paper proposes and implements an automatic power line extraction method based on 3D spatial features. Different from the existing power line extraction methods, the proposed method processes the LiDAR point cloud data vertically, therefore, the possible location of the power line in point cloud data can be predicted without filtering. Next, segmentation is conducted on candidates of power line using 3D region growing method. Then, linear point sets are extracted by linear discriminant method in this paper. Finally, power lines are extracted from the candidate linear point sets based on extension and direction features. The effectiveness and feasibility of the proposed method were verified by real data of UAV LiDAR point cloud data in Sichuan, China. The average correct extraction rate of power line points is $98.18 \%$.
\end{abstract}

\section{INTRODUCTIONS}

Power line is the essential infrastructure for most of our social economic activities. Monitoring power line efficiently and effectively is of crucial importance, especially in power line risk management. Accurate knowledge of the status about the power line and the possible dangerous points is one of the key tasks in power line management. The traditional inspection and maintenance of power line mainly rely on manual field survey, which is financially costly and operationally inefficient. Moreover, field crew also face various safety issue in the field. An efficient and flexible surveying scheme is needed to overcome this problem. In 1950s, helicopters equipped with digital cameras, infrared cameras, etc., were used for the power lines inspection and maintenance. However, these technologies are limited by low positioning accuracy, and the data processing is complex (Cheng, 2004). Airborne LiDAR system can solve the problems of poor spatial positioning and measurement precision (Xu, 2017). Those systems acquire 3D point cloud data with high precision along power line corridor, as well as obtain spatial information of the power line corridor terrain and line facilities. Thus, airborne LiDAR measurement technology was applied widely in power line inspection (You, 2013). The methods for power line detection based on airborne LiDAR point cloud data can be summarized into two categories: Linear feature detection methods and supervised classification methods.

Based on linear feature detection, point cloud data constructions and features analysis methods are common way for power lines extraction. Axelsson (Axelsson, 1999) separated the power line point cloud data from the vegetation using multi echoes and intensity information, then the line structure is further detected using Hough transform. Melzer and Briese (Melzer, 2004) extracted the power lines by an iterative version of the Hough transform from the point cloud data after filtering, which can only be used for the power line extraction in small areas. Clode and Rottensteiner (Clode, 2005) could distinguish power lines and trees by analysing the intensity and elevation differences between the first and last echoes, but the echo intensity values should be calibrated and the method lack adaptation. Yuee Liu et al. (Liu, 2009) distinguished the ground and non-ground points by statistical analysis of the skewness and kurtosis of the intensity for the LiDAR point cloud data, and used Hough transform to detect the power lines from the point cloud data after filtering Jwa and Sohn (Jwa, 2009) proposed a power line extraction method based on LiDAR point cloud data using three constraint conditions of power line height, direction and power tower position, then the power lines were detected by Hough transform. Ye et. al. (Lan, 2010) obtained elevation images by elevation projection and resampling, then extracted and fitted the power lines in the image space by line detection. Yu (Jie, 2011) adopted LiDAR point cloud data filtering method to remove the ground points and vegetation points, and then used two-dimensional Hough transform to separate the power lines, finally, a single power line was fitted according to the hyperbolic cosine function. However, the extraction results were poor in the regions, which were mixed by trees and power lines. Zhu and Hyyppä (Zhu, 2014) proposed an automated power line detection method based on statistical analysis and 2D image-based processing technology. During the process of statistical analysis, a set of criteria (e.g., height criteria, density criteria and histogram thresholds) was applied for selecting the power lines candidates. After transforming the candidates to a binary image, image-based processing technology was employed. Object geometric properties were considered as criteria for power line detection. Liu et al. (Liu, 2014) proposed and implemented a method for automatic extraction of power lines from LiDAR point cloud data based on spatial domain segmentation. Firstly, the method

\footnotetext{
* Corresponding author
} 
removed the ground points, and then used the differences of point cloud data density to eliminate the power poles. The single power line was separated according to the distance differences between adjacent lines and the elevation differences between adjacent layers. Finally, the 3D coordinate was reconstructed for each power line based on polynomial model. However, for the complex urban environment data, this method were not ideal, vegetation and building point cloud data should be further removed manually. Lin (Lin, 2016) extracted the accurate power pole location and number, power line track and other information according to pre-knowledge of the power pole position and the initial power line track data. After power line central projecting, $\mathrm{K}$-means clustering was used to divide each power line point cloud data into the corresponding line, however these methods were only verified on power lines between two power poles, long distance power line extraction was still difficult. The mentioned methods can all extract the power line point cloud data, but most of them need to filter the point cloud data. And the methods based on Hough transform cannot sense multiple power lines in vertical arrangement, however 3D Hough can solve the issues mentioned above.

Power line extraction methods based on supervised classification were also presented. Robert (Mclaughlin, 2006) presented an algorithm to extract high-voltage power lines using airborne LiDAR data. LiDAR data was first labelled as power line, vegetation and surface using Gaussian mixture model. The extracted points of power line were then segmented into individual spans using local affine models. However, only $72.1 \%$ of the individual power line spans were extracted. Kim (Kim, 2013) proposed a point-based supervised classification method for power line extraction in airborne LiDAR data. The method enabled the identification of five utility corridor objects (wires, pylons, vegetation, buildings and low objects). A total of 21 features were investigated to illustrate the horizontal and vertical properties of power line. Then, a non-parametric discriminative classifier, i.e. random forests, was trained with refined features to label raw laser point cloud data. The proposed classifier showed more than $90 \%$ classification accuracy. However, the classifier produced classification errors that required manual editing. Guo (Guo, 2013) proposed a supervised classification method to identify power lines, pylons, buildings, ground and vegetation from point cloud data using a JointBoost classifier. The JointBoost classifier was modeled with various features obtained from LiDAR point cloud data. The overall accuracy was more than $95 \%$. Wang et. al (Wang, 2017) presented a novel fully automated and versatile framework based on Support Vector Machines (SVM) classification. In a detailed evaluation involving seven neighbourhood definitions and 26 geometric features with two datasets, they demonstrated that the use of multi-scale neighbourhoods for individual 3D points is able to significantly improve power line classification accuracy. For supervised classification methods with multiple features, large training dataset and computational time are required to achieve the desired results. Furthermore, different models are needed to be trained for specific study areas.

In the last few years, UAV (Unmanned Aerial Vehicle) applications in the civil field have become increasingly popular. Their use is becoming widespread in many scientific disciplines (Capolupo, 2015). Moreover, their large market penetration and continuous development have led to a drastic reduction in their cost (Capolupo, 2014). In practice, most of the published studies about power line extraction based on UAV data have used optical images, and many of them have concentrated on line feature detection methods (Matikainen, 2016), which cannot sense multiple power lines in vertical arrangement. With the recent technical electronic and optical improvements for aerial platforms and the devices mounted on them, LiDAR system can be mounted on the UAVs. The power line extraction based on UAV LiDAR data mostly used the above linear feature detection methods or supervised classification methods. Therefore, this paper presents an automatic extracting power line method from UAV LiDAR data based on 3D spatial features of the power lines, which does not need point cloud filtering or training classification models. The proposed method extracts power line by considering the spatial features of point clusters of different classes only. In this method, the point cloud data are processed from top to bottom vertically without point cloud data filtering. The 3D region growing method is used for segmentation of power line candidates. Then, linear point sets are extracted by linear discriminant method. Finally, the points of power lines are extracted from the linear point sets according to the horizontal extension and direction features. The test results show that the proposed method is easy to be implemented, and efficient at high degree of automation. Moreover, the proposed method is effective to point density and topographic variations.

This paper proposes and implements an automatic power line extraction method based on 3D spatial features using UAV LiDAR point cloud data. The contents of the paper are organized as follows. Section 2 detailed the experimental material. The proposed method was introduced in Section 3. Experiments results and discussions were presented in section 4. Section 5 drew the conclusions.

\section{MATERIAL}

The experimental LiDAR data in this paper was acquired in Xichang, Sichuan Province by AOEagle, which is developed by Academy of Opto-electronics, Chinese Academy of Sciences (Teng, 2017). The AOEagle LiDAR system is equipped on a UAV of six rotors, as shown in Figure 1. Main technical parameters of AOEagle are listed in Table 1. The absolute flight height of this experiment is from $2800 \mathrm{~m}$ to $3000 \mathrm{~m}$ (above ground varied between $50 \mathrm{~m}$ and $150 \mathrm{~m}$ ).

Data description is given in Table 2. The method was tested on all the 6 data sets. Especially, in data set 6, poles, power lines and ground points distribute uniformly. Moreover, both straight line poles and corner poles present in data set 6 , as shown in Figure 4. Therefore, this work takes data set 6 as a benchmark to elaborate the processing and the extraction results from the other five data sets are compared. In order to analyse the effects of the average point density, the data 6 was thinned to be data 5 just as listed in Table 2.

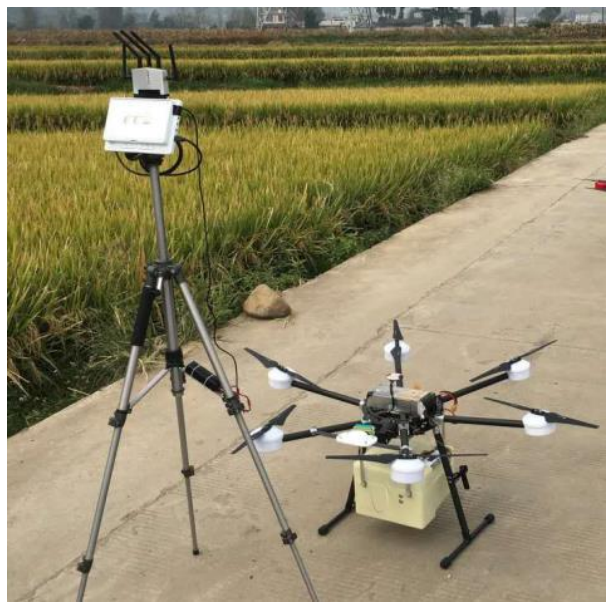

Figure 1. AOEagle is equipped on a UAV of six rotors. 


\begin{tabular}{|l|c|}
\hline Item & Description \\
\hline Weight & $3.5 \mathrm{~kg}$ \\
Power Consumption & $13 \mathrm{~W}$ \\
Angular Resolution & $0.125^{\circ}$ \\
Maximum Range & $200 \mathrm{~m}$ \\
Distance & $110^{\circ}$ \\
Detection Angle & $4 \mathrm{~cm}$ \\
Range Resolution & $905 \mathrm{~nm}$ \\
Wavelength & $0.08^{\circ}$ \\
Azimuth Accuracy & $0.015^{\circ}$ \\
Pitch Accuracy & $0.015^{\circ}$ \\
Roll Accuracy & $2 \mathrm{~cm}$ \\
Horizontal Accuracy & $2 \mathrm{~cm}$ \\
Vertical Accuracy & \\
\hline
\end{tabular}

Table 1. Specifications of the AOEagle

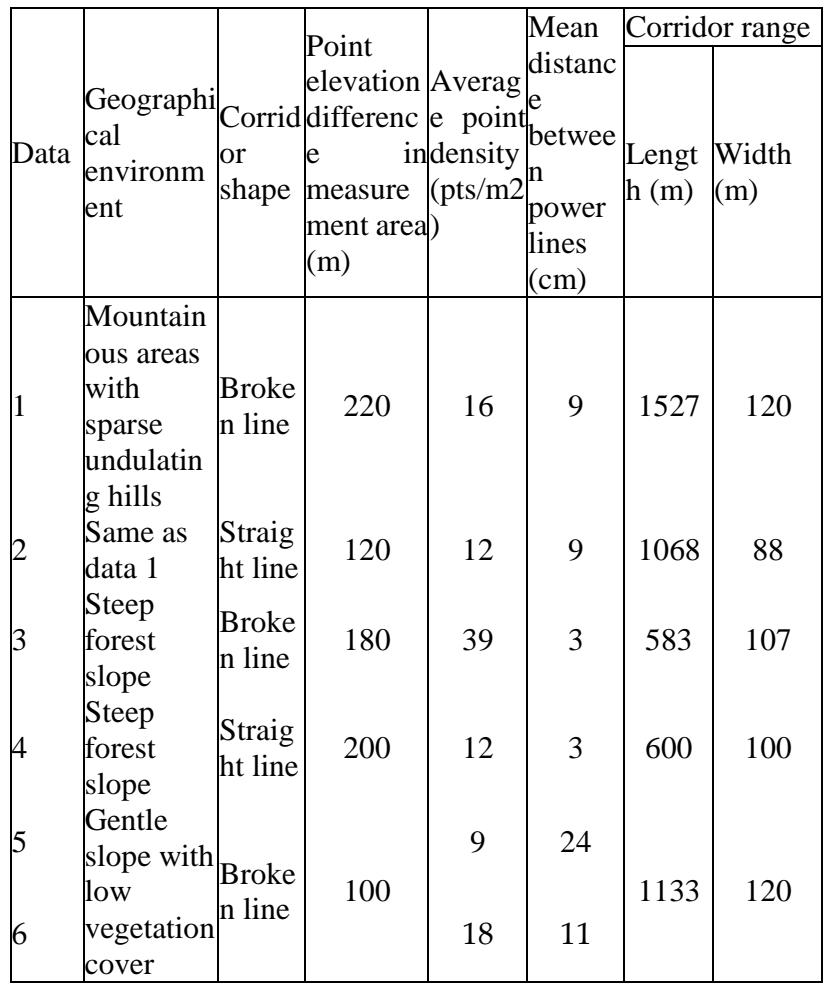

Table 2. Data characteristics of each study area.

\section{METHOD}

\subsection{Characteristics of power line point cloud data}

To extract power lines from LiDAR point cloud data, it first needs to analysis the features of power lines in point cloud data. The next step is to separate points of power lines from huge LiDAR point cloud data, which means to identify the power lines. The features of power lines in point cloud data include:

1. Power line is always at the top of the corridor, and there are no other objects above it;

2. There is a certain standard for the distance between power lines; the distance between the ground surface and the lowest layer of the power line is bigger than that between each power lines;

3 . The power lines are linearly distributed through the entire region, and have a strong extension and direction feature in horizontal direction;

4. Elevation of a single power line is similar in a small area.

\subsection{Overall work-flow of power line extraction}

Firstly, outliers are removed from the original point cloud data by pre-processing. Secondly, parameters are setup according to the characteristics of the power lines. Thirdly, the power line candidates are selected according to elevation. Fourthly, the candidate points are divided into different point sets, which are called elements in this paper, and then the linear elements are extracted. Finally, power line elements were identified from the elements according to the 3D spatial characteristics of power lines. The overall work-flow of power line extraction method in this article is shown in Figure 2.

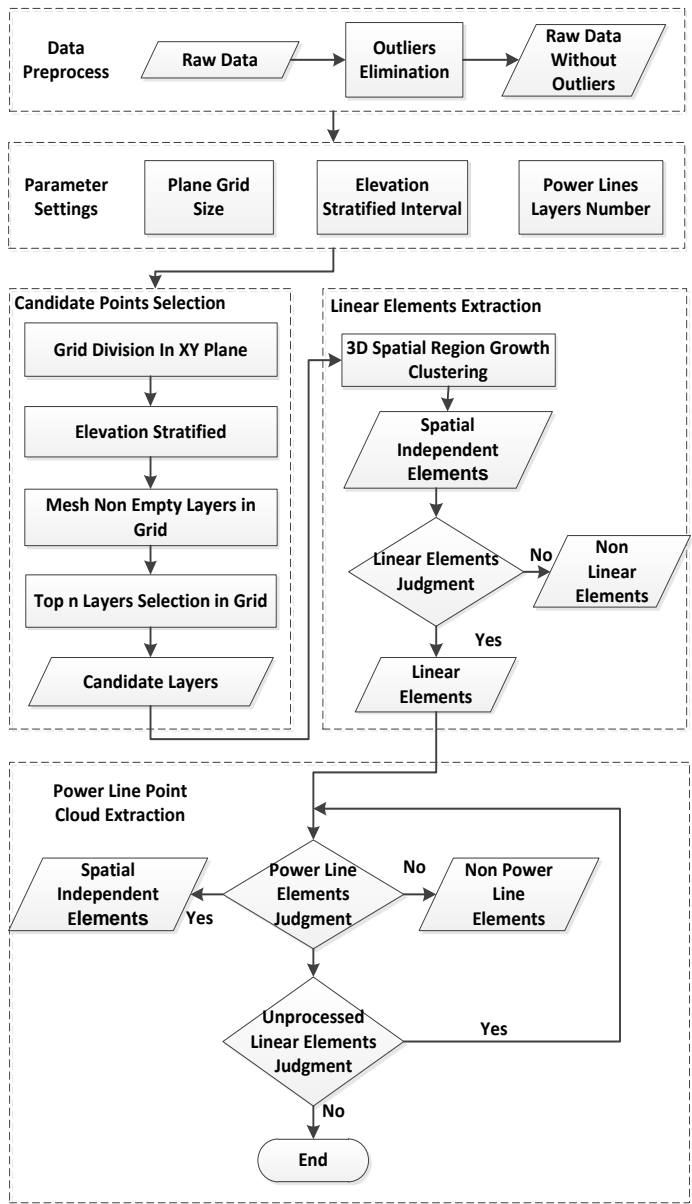

Figure 2. Overall work-flow of the proposed power line extraction method.

\subsection{Pre-processing and parameter settings}

Due to circuits, birds, fog and other reasons, the abnormal distance value above the power line will have a great impact on the data processing results in this paper. Therefore, the original point cloud data needs to be pre-processed to exclude the abnormal points with the high-altitude in the original point cloud data. According to the density of the laser point cloud and the horizontal distance $D_{x}$ between each wire, the size of $m$ in X-Y plane is set. The parameter $\mathrm{m}$ is calculated by formula (1).

$$
m<\frac{D_{x}}{2}
$$

where $\quad m=$ size of grid in $\mathrm{X}-\mathrm{Y}$ plane

$D_{x}=$ horizontal distance between each wire

The elevation stratified $d$ is determined according to the vertical distance $\Delta h$ between each power line, as shown in Figure 3. In 
order to make the adjacent power line layer divided into different layers, the calculation formula of $\mathrm{d}$ is shown as formula (2).

$$
d<\frac{\Delta h}{2}
$$

where

$d=$ elevation stratified

$\Delta h=$ vertical distance between each power line

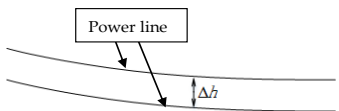

Figure 3. Vertical distance between power lines.

As shown in Figure 4, in the study area of data 6, the topographic relief is small and there is no tall tree, average point density is 18 $\mathrm{pts} / \mathrm{m} 2$. According to the relevant specifications of power line construction and the speed of data processing, the planar grid size is set as 3 to 5 meters. The results are good when the grid size $\mathrm{m}$ is 3 meters and height layer spacing $\mathrm{d}$ is 4 meters. Based on the practical condition, the power line is vertically divided into two layers, so we set $n_{f}$ to be 2 . Unless otherwise specified, the illustrations are colored according to the height.

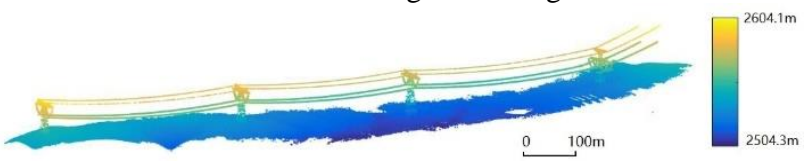

Figure 4. Point cloud of power line corridor in gentle slope topography. The colour bar represents the real height range.

\subsection{Determination of power line candidates}

According to the main features of the power line in LiDAR point cloud data, the data is processed from top to bottom in vertical direction and gridded in the XY plane. Then, stratifying the point cloud data in each grid based on the distance $\Delta \mathrm{h}$ between the adjacent layers. Meanwhile, merging the non-empty layers to make that each grid point cloud data are contained in several independent layers. According to the real power line layers number, $n_{f}$ power line candidate layers are selected from top to bottom in each grid. Specific steps are listed below:

\section{Gridding in XY plane}

Point cloud data is resampled to grids with grid size $\mathrm{m}$ and the indices of each point in the grids were established.

2. Vertical stratification and merging in each grid

The maximum elevation $z_{\max }$ and minimum elevation $z_{\min }$ in each grid were identified, which could help calculate the elevation difference of the point cloud data, then each grid was divided into Num layers in the vertical direction with $d$ as the interval threshold.

$$
\text { Num = round }\left\lceil\frac{Z_{\max }-Z_{\min }}{d}\right\rceil
$$

where $\quad z_{\max }=$ maximum elevation value

$z_{\min }=$ minimum elevation value

$d=$ elevation stratified

round $\lceil\quad$ = upward rounding function

Using an iterative approach to divide the point cloud data into the corresponding cubic cells. Then, determine whether the two adjacent layers are empty in the grid. If both of layers are not empty, merge them into one layer. Then determine whether the new layer adjacent layer is empty, to achieve all non-empty adjacent layer of the merger. Finally, each grid gets a number of independent layers of the space. The elevation stratificationmerge diagram is shown in Figure 5.

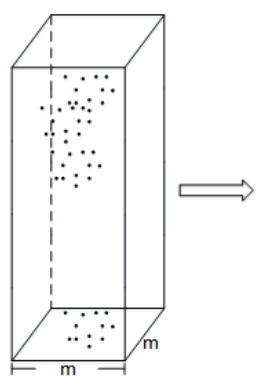

(a)

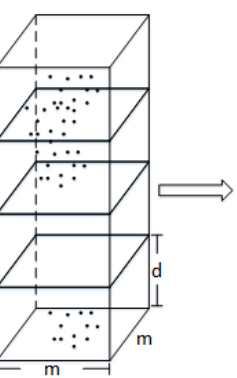

(b)

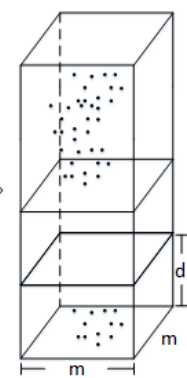

(c)
Figure 5. Elevation stratification-merge diagram. (a) Raw Point Cloud data; (b) Stratification; (c) Merge of the non-empty cells.

3. Selection of power line candidate layers

The power line is located on the top of point cloud data, for the reason that the power line is catenary, and the power line diameter is less than the point cloud data layer elevation difference. So the top $n_{f}$ layers of each grid are selected to calculate the elevation difference of each layer, if the difference is not more than $h$ this one is a candidate layer; if the difference is greater than $h$, there is no power line layer in this grid. Due to the curvature of each wire are varied, considering the applicability of the $h$ in the whole wire, we use the maximum curvature point to calculate $h$.

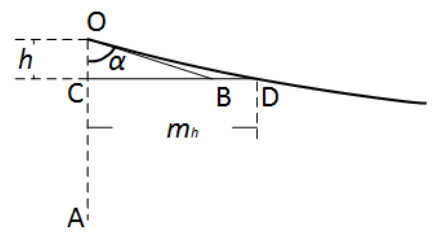

Figure 6. Calculation of the elevation difference of each layer. As shown in Figure 6, h is determined by formula (4), as given below.

$$
h=\frac{m_{h}}{\tan \alpha}, m_{h} \in[m, \sqrt{2} m], \alpha \in\left[\frac{\pi}{4}, \frac{\pi}{2}\right)
$$

where $\quad h=$ threshold value of power line layer

$\alpha=$ angle between power line tangent and vertical direction

$m_{h}=$ projected length of the power line in the grid horizontally

In the study area of the data 6 , based on the parameters settings in section 3.3, the presented method first grids the whole power line corridor. The point cloud data is divided into the corresponding horizontal square grid, and then is divided into the 3D grid with 4 meters intervals. Different 3D grid represents different point cloud data layers, by merging non-empty adjacent 3D grids, two continuous point cloud data layers are combined into one layer. The top two layers of each grid are selected as candidates, it can be seen in Figure 7. The candidate points contain almost all of the power line points, part of the bare ground and vegetation points, and remove most of the pylon points.

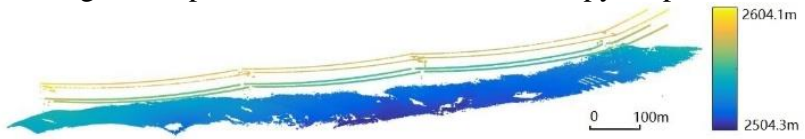

Figure 7. Power line candidates. The color bar represents the real height range. 


\subsection{Extraction of linear elements}

In different grids, a region growing method based on 3D space is used to cluster the candidate layers, and the independent sets of points in the whole measurement area are obtained. Then, the linear elements can be obtained by the width features of the elements projection in the XY plane. Specific implementation methods are as following:

1. Clustering based on 3D spatial region growth

According to the region growing method in 3D space, for each grid, each of its 8 neighbourhood grids were searched first. Then, average elevations of points in central grid and 8 neighbourhood grids were calculated respectively. Meanwhile, if the average elevation difference in central grid layer and any layer of a neighbourhood grid is smaller than $\mathrm{h}$, these two layers were clustered into one layer. The clustering of power candidate layers in all grids was traversed iteratively which results in multiple spatially independent elements.

\section{Linear element extraction}

According to the coordinates of each point in every element, their 8 neighbourhood grids were first acquired. Then, the diameter of the inscribed circle in horizontal plane that was projected from points in central grid and its 8 neighbouring grids was calculated. Considering measurement error and accuracy, we chose a $D_{r}$ as a criterion, which was slightly larger than the power line diameter $D_{\rho}$. If the inscribed circle diameter value is greater than $D_{r}$, this element belongs to nonlinear element; otherwise, it belongs to linear element. The calculation of the diameter of the inscribed horizontal circle from point cloud data is shown in section 3.6. The main projection types of elements are shown in Figure 8.

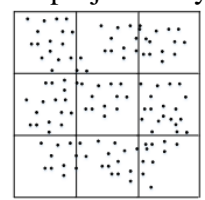

(a)



(b)

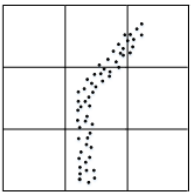

(c)
Figure 8. Sketch of surface and linear projection. (a) Planar; (b) Linear; (c) Polygonal.

According to the region growing method in 3D space, several independent elements can be obtained by clustering the point cloud data of candidate layers. In this paper, we set $D_{r}$ as $3.8 \mathrm{~m}$ to determine the linear element based on the above criterion. Figure 9 shows the results of the linear element extraction from power line candidates. The results show that the power line point cloud data was well segmented and most of the non-power line point cloud data was eliminated.

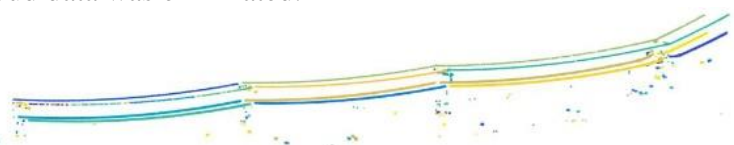

Figure 9. Linear element extraction result (colored by element).

\subsection{Extraction of power line point}

The points of power line are linearly distributed and have dominant horizontal directional extension. Therefore, power line elements can be identified by the directional extension and linear characteristics. In this paper, the directional extension of linear elements is described by calculating the diameter of the inscribed/circumscribed horizontal circles of the projected points. The diameter of the inscribed/circumscribed circles is equal to the diameter and length of the linear element. The diameter of the circumscribed circle describes the extensional characteristics, which can be used to judge whether the linear element has obvious directional by the diameter ratio of the circumscribed circle to the inscribed circle. The example of the inscribed/circumscribed circles is shown in Figure 10. The point $p$ is the center of the circle. The line segment $p q$ is the radius of inscribed circle, whose length is $r$. The line segment $p Q$ is the radius of a circumscribed circle, whose length is $R$. The implementation workflow is given below:

1. Calculate the center of gravity of each point in the element in the XY plane $\mathrm{p}$ (xMean, yMean), and take it as the common center of the inscribed and circumscribed circles;

2. Firstly, the distances between all the points and $p$ of each element are calculated. Then, the circumscribed circle radius $\mathrm{R}$ was set to the maximum distance. Supposing the point which has the largest distance to point $\mathrm{p}$ is $\mathrm{Q}$, Calculate the directional vector $R_{v}$ of $\mathrm{pQ}$, then calculate the direction vector $r_{v}$ that perpendicular to $\mathrm{pQ}$ according to $\mathrm{R}_{\mathrm{v}}$, and take the $r_{v}$ as the direction vector to determine the linear equation $\mathrm{AX}+\mathrm{BY}+\mathrm{C}=0$;

3. The buffer zone on both sides of the line determined in step 2 was set, if the distance between the point and the line is not bigger than $D_{f}$, which is half of the width of the buffer and associated to the point spacing, then this point is regarded as the candidate of inscribed circle edge point; if there is no point in the buffer, then $D_{f}$ is increased until a point locates at half of the width of the buffer on both sides of the line; Next, compute the distance between candidate edge points and point $p$, then set the maximum distance as the radius of inscribed circle of $r$. If $2 r>D_{r}$, this linear element is polygonal, then the value of $D_{r}$ is the diameter of the inscribed circle; if $R / r>v_{f}$, this element has obvious directional characteristics, so we classify it to power line element. Meanwhile, $\mathrm{v}_{\mathrm{f}}$ is a variable threshold, whose value should be more than 2 times the length of the plane grids.

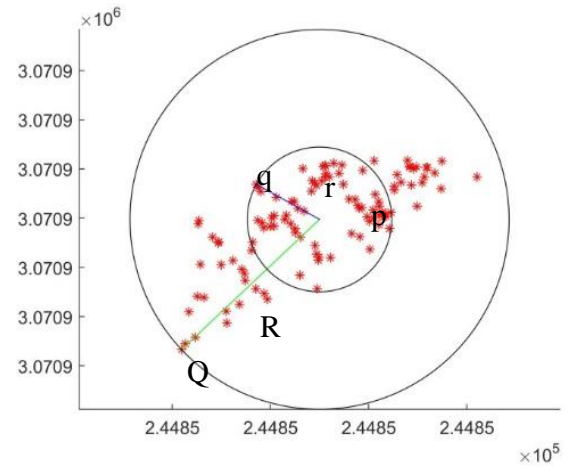

Figure 10. Example diagram of inner and outer circles.

In order to eliminate ground fragmentation, as shown in Figure 9, a suitable $v_{f}$ is needed. Figure 11 shows the power line extraction results when $v_{f}$ is set to 4 . From the figure we can see that, when the $v_{f}$ is 4 , the power line element cannot be separated from other linear element well, just as the red marks in Figure 11. When $v_{\mathrm{f}}$ is 8 , only the power point cloud data is left in the extracted result, as shown in Figure 12. It can be seen that the result is the best when the value of $v_{f}$ is more than 2 times of the grid length.

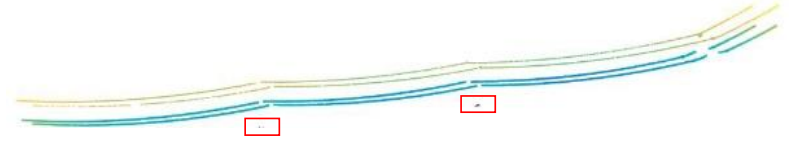

Figure 11. Power line points extraction results when $v_{f}=4$. 
Figure 12. Power line points extraction results when $\mathrm{v}_{\mathrm{f}}=8$.

It can be seen that the power line point cloud data in data 6 were almost extracted in Figure 12. We used the same method to process the other five data sets, the results are listed in Figure 13.

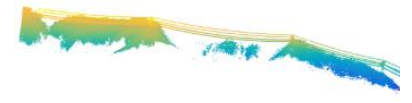

Data 1

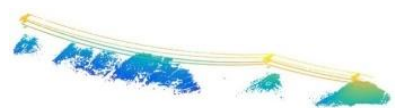

Data 2

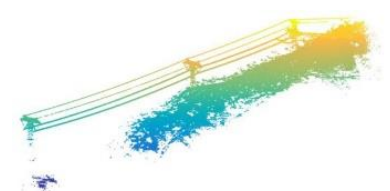

Data 3

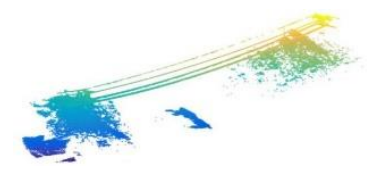

Data 4

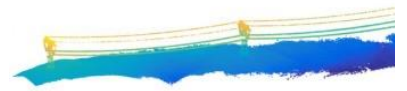

Data 5
Data 1 Results

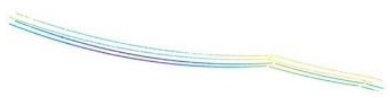

Data 2 Results

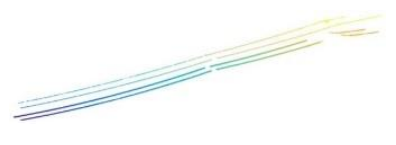

Data 3 Results

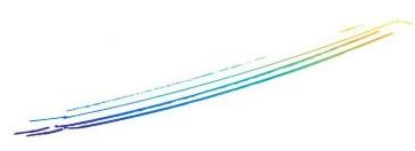

Data 4 Results

Data 5 Results
Figure 13. Processing results of the remaining 5 sets of data.

\section{RESULTS AND DISCUSSIONS}

To verify the feasibility and reliability of the proposed method, data sets contain different terrains, pole types and point cloud data density were selected as shown in Table 1 . Combined with the characteristics of this algorithm, we also analysed the influences of power line spacing on extraction results.

We analysed the power line extraction rate of each data sets based on manual extraction results that are considered as the true values in this paper. As shown in Table 2, the average extraction rate (i.e., the percentage of correctly identified power line points to entire number of points.) is $98.18 \%$. The terrain in the 6 sets of data is complex and diverse. However, the power line extraction rate has reached $95 \%$, which means the results of the proposed method are not affected by the measurement area terrain.

\begin{tabular}{|c|c|c|c|c|c|c|}
\hline \multirow[b]{2}{*}{$\begin{array}{c}\text { Dat } \\
\text { a }\end{array}$} & \multirow{2}{*}{$\begin{array}{c}\text { Avera } \\
\text { ge } \\
\text { point } \\
\text { densit } \\
\text { y } \\
\text { (pts/m } \\
\text { 2) }\end{array}$} & \multirow{2}{*}{$\begin{array}{c}\text { Mean } \\
\text { distanc } \\
\mathrm{e} \\
\text { betwee } \\
\mathrm{n} \\
\text { power } \\
\text { lines } \\
(\mathrm{cm}) \\
\end{array}$} & \multicolumn{2}{|c|}{$\begin{array}{c}\text { Power line points } \\
\text { number }\end{array}$} & \multirow[b]{2}{*}{$\begin{array}{l}\text { Extracti } \\
\text { on rate }\end{array}$} & \multirow[b]{2}{*}{$\begin{array}{c}\text { Average } \\
\text { extractio } \\
\mathrm{n} \text { rate }\end{array}$} \\
\hline & & & $\begin{array}{c}\text { Automat } \\
\text { ic } \\
\text { extractio } \\
n\end{array}$ & $\begin{array}{c}\text { Manual } \\
\text { extractio } \\
n\end{array}$ & & \\
\hline 1 & 16 & 9 & 75541 & 76806 & $98.35 \%$ & \\
\hline 2 & 12 & 9 & 51452 & 52181 & $98.60 \%$ & \\
\hline 3 & 39 & 3 & 71774 & 75180 & $95.47 \%$ & \\
\hline 4 & 12 & 3 & 68702 & 70111 & $97.99 \%$ & $90.10 \%$ \\
\hline 5 & 9 & 24 & 23052 & 23232 & $99.23 \%$ & \\
\hline 6 & 18 & 11 & 45597 & 45336 & $99.43 \%$ & \\
\hline
\end{tabular}

Table 3. Statistics of power line extraction results.

Since the average point spacing distributed uniformly and the size of it was less than the plane grid, thus it is easy to clustering power line point cloud data into every one element. It can keeps the power line element extensibility and direction characters pretty well, even if the average density is different, the extraction rate is stable, just as data 1 , data 2 , data 5 and data 6 .

Data 2 and data 4 belong to linear power line, but the extraction rate of data 4 was less than that of the data 2 by $0.61 \%$. The reasons could be: as shown in Figure 14, the data 4 power line point cloud data has several discontinuous segments, the lack distance of adjacent segments was greater than 6 meters. Therefore, in the process of region growing, the points in the block could not be clustered as the same element with the left point cloud data. Instead, they were clustered into several independent elements as shown in Figure 15. When extracting the power line elements, the horizontal extension features of these independent elements do not meet the requirements of the power line element, resulted in the lack of power line point cloud data in the block. While the point spacing in data 2 is welldistributed, only a few discontinuous segments existed, and the missing distance of the adjacent segment is less than 3 meters, so power lines in data 2 were well extracted.

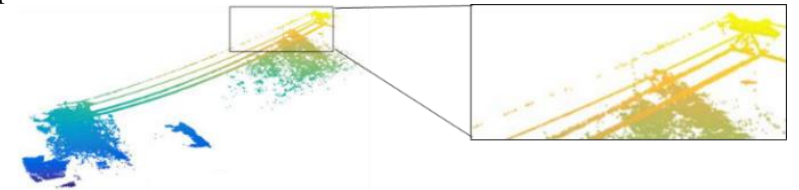

Data 4

Enlarged drawing of Data 4

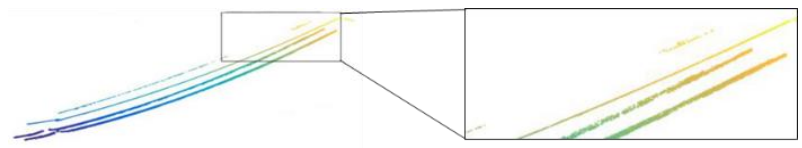

Results of Data 4 Enlarged drawing of the results of Data 4

Figure 14. Partial enlarged drawing for Data 4 and its result 


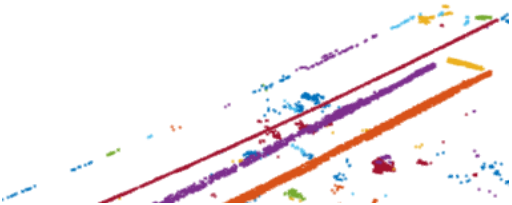

Figure 15. Linear elements extraction results for the partial enlarged part of Data 4 (coloured by element).

The extraction results for data 3 has the lowest success rate, mainly due to the existence of corner pole as shown in Figure 16. The insulators on the corner pole are approximately straight in horizontal direction, which causes the unsuccessful extraction of the power lines pass through the insulators. The average point density of data 3 is the highest, which makes the absence more obvious.

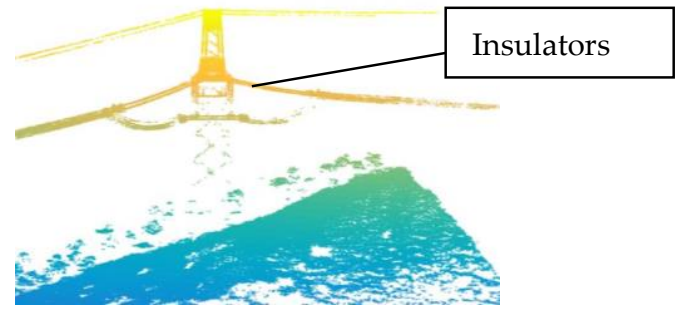

Figure 16. Corner tower.

Compared with the data 3 , the data 2 belongs to the linear power line corridor and the insulators are vertically installed, as shown in Figure 17. The extraction of power lines only removed the points in the grid with the hanging point, so the effect of the insulator was smaller, therefore the extraction effect reached $98.60 \%$.

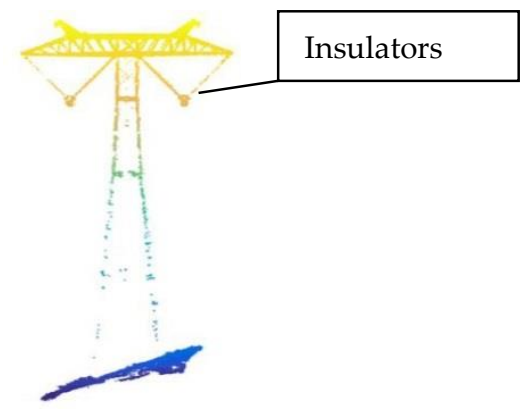

Figure 17. Straight pole.

Data 5 was obtained after thinning data 6. Compared to the data 6 , the power line point extraction rate of data 5 is only dropped by $0.2 \%$. Although the average power line spacing in data 5 and 6 is the largest, the power lines extraction results for data 5 and 6 are the best. The reason is that the power line spacing of the two data sets is uniform and less than the size of the plane grid. We could find that when the point spacing of the power line is smaller than the size of the plane grid, the power line points can be extracted and the extraction effect is not restricted by the average point density in the measurement area.

\section{CONCLUSIONS}

Based on analysis of main characteristics of the power line point cloud data in 3D space, this paper proposes an automatic power line extraction method from UAV LiDAR point cloud data based on the 3D spatial features of power line, which can effectively extract the power line point cloud data from the LiDAR point cloud data. The proposed method processed the point cloud data vertically; therefore, the possible location of the power lines in point cloud data can be predicted without point cloud data filtering. The data processing flow is simple, and the method is not affected by the terrain and the average point density in the measurement area. Moreover, automatic extraction of power lines between multiple towers is realized with high precision. The experiment shows that the method has a good extraction rate on the straight / polygonal power lines corridor, and the average extraction rate of the 6 sets of power lines is up to $98.18 \%$, which is according to the comparison with the manual extraction results. But for the corner poles, it cannot extract the power lines below insulator, the method needs to be further improved.

\section{ACKNOWLEDGEMENTS}

The work is supported by the [CAS Pioneer Hundred Talents Program] under Grant [No. Y960120Z1F].

\section{REFERENCES}

Axelsson, P., 1999. Processing of laser scanner data—algorithms and applications. ISPRS Journal of Photogrammetry \& Remote Sensing, 54(2-3), 138-147.

Cheng, Z.F., Liang, Q.Y., 2004. The application of airborne laser scanning imaging technology in transmission line survey. Electric Power Survey. 1671-9913(2004) 01-0031-05

Clode, S., Rottensteiner, F., 2005. Classification of trees and power lines from medium resolution airborne laser scanner data in urban environments. In: Proc. APRS Workshop on Digital Image Computing (WDIC 2005), Brisbane, Australia, 21 February, 2005.

Capolupo, A., Pindozzi, S., Okello, C., Boccia, L., 2014. Indirect field technology for detecting areas object of illegal spills harmful to human health: application of drones, photogrammetry and hydrological models. Geospatial Health, 2014, 8(3), S699.

Capolupo, A., Kooistra, L., Berendonk, C., Boccia, L., Suomalainen, J., 2015. Estimating plant traits of grasslands from uav-acquired hyperspectral images: a comparison of statistical approaches. ISPRS International Journal of Geo-Information, 2015, 4(4), 2792-2820.

Guo, B., Huang X.F., Zhang F., 2013. Points Cloud Classification Using JointBoost Combined with Contextual Information for Feature Reduction. Acta Geodaetica et Cartographica Sinica, 42(5), 715-721.

Jwa, Y., Sohn, G., Kim, H.B., 2009. Automatic 3d powerline reconstruction using airborne lidar data. Iaprs, (2014):2009.

Jwa, Y., Sohn, G., 2010. A multi-level span analysis for improving $3 \mathrm{~d}$ power-line reconstruction performance using airborne laser scanning data. ISPRS Technical Commission III Symposium PCV 2010 - Photogrammetric Computer Vision and Image Analysis, France, September 2010.

Jie, Y.,Mu, C., Feng, Y., Dou, Y., 2011. Powerlines extraction techniques fromairborne lidar data. Geomatics \& Information Science of Wuhan University, 36(11),1275-1279.

Kim, H.B., Sohn, G., 2013. Point-based classification of power line corridor scene using random forests. Photogrammetric Engineering \& Remote Sensing, 79(9), 821-833.

Liu, Y., Li, Z., Hayward, R., Walker, R., Jin, H., 2009. Classification of Airborne LIDAR Intensity Data Using Statistical Analysis and Hough Transform with Application to 
Power Line Corridors. Digital Image Computing: Techniques and Applications , Melbourne, Australia, 1-3 December, 2009.

Lan, Y. E., Liu, Q., Qing-Wu, H. U. 2010. Research of power line fitting and extraction techniques based on lidar point cloud data. Geomatics \& Spatial Information Technology., 33(5),30-34.

Liu, Z., Jing, L., Zhang, J., 2014. Power lines extraction from airborne lidar data using spatial domain segmentation. Journal of Remote Sensing, 1, 61-76.

Lin, X., Zhang, J., 2016. 3d power line reconstruction from airborne lidar point cloud of overhead electric power transmission corridors. Acta Geodaetica Et Cartographica Sinica.

Melzer, T., Briese, C., 2004. Extraction and modeling of power lines from als point clouds. Proceedings of Workshop.

Mclaughlin, R.A., 2006. Extracting transmission lines from airborne lidar data. IEEE Geoscience \& Remote Sensing Letters, $3(2), 222-226$.

Matikainen, L., Lehtomäki, M., Ahokas, E., Hyyppä, J., Karjalainen, M., Jaakkola, A., 2016. Remote sensing methods for power line corridor surveys. ISPRS Journal of Photogrammetry \&Remote Sensing, 119, 10-31.

Teng, G.E., Zhou, M., Li, C.R., Wu, H.H., Li, W., Meng, F.R., Zhou, C.C., Ma, L., 2017. Mini-UAV LiDAR for power line inspection. The International Archives of the Photogrammetry, Remote Sensing and Spatial Information Sciences, XLII-2/W7, 297-301.

Wang, Y., Chen, Q., Li, K., Zheng, D., Fang, J., 2017. Airborne LiDAR power line classification based on spatial topological structure characteristics. ISPRS Annals of the Photogrammetry, Remote Sensing and Spatial Information Sciences, IV-2/W4, 165-169.

Xu, L., Xu, T., Wang, J., Li, X., 2017. A fuzzy pid controllerbased two-axis compensation device for airborne laser scanning. IEEE Sensors Journal, 17(5), 1353-1362.

Xu, T., Xu, L., Tian, X., Li, X., 2017. Gps-aided method for platform attitude determination based on target images. Applied Optics, 56(8), 2378.

You, A.Q., Wang, X.P., Han, X.Y., Tang, D., 2013. Applications of LiDAR in patrolling electric-power lines. International Conference on Technological Advances in Electrical, Electronics and Computer Engineering, Turkey, 9-11 May 2013.978-14673-5613-8

Zhu, L., Hyyppä, J., 2014. Fully-automated power line extraction from airborne laser scanning point clouds in forest areas. Remote Sensing, 6(11), 11267-11282. 